\title{
Las parasitosis intestinales más frecuentes en la población infantil del área de salud de San Ramón de Alajuela
}

\author{
Erasmo Serrano-Frago, ${ }^{1}$ Adriana Cantillo-Arrieta ${ }^{2}$
}

\begin{abstract}
Justificación y Objetivo: Las parasitosis intestinal por helmintos y protozoarios continuan teniendo gran impacto en la salud de muchas poblaciones alrededor del mundo. El presente es un estudio para determinar la frecuencia de dichas parasitosis en niños de 1 a 12 años en el área de salud de San Ramón de Alajuela, Costa Rica.
\end{abstract}

Método: Estudio retrospectivo en el que se analizaron muestras de heces de 5250 niños de 1 a 12 años de diciembre de 1999 a noviembre de 2000. Esta población representa cerca de un 29\% de la población infantil de 1 a 12 años de la zona, de acuerdo con la población estimada según los compromisos de gestión. Las muestras de heces fueron solicitadas por los médicos tratantes por sospecha de parasitosis intestinal, y a las mismas se les realizó frotis y extendidos simples con la técnica de solución salina y lugol. Se completaron los datos a través de una revisión de expedientes médicos.

Resultados: En total un $40.4 \%$ de la población estudiada presentó parasitosis intestinal. Se presentaron 1478 casos de protozoarios en las muestras de heces y 645 casos presentaron hallazgo de helmintiasis. Dentro de los protozoarios los más frecuentemente encontrados fueron E. histolytica, E. coli y G. intestinalis. Entre las helmintiasis predominaron la E. vernicularis, S. stercoralis y H. nana. Del total de niñas, $30.7 \%$ presentaron parasitosis intestinal contra un $49.7 \%$ de los varones.

Conclusión: El presente estudio evidencia una disminución en la frecuencia de helmintiasis, la cual seguramente obedece a las campañas masivas que se han hecho, mientras que el incremento en los protozoarios probablemente se deba a que no se ha tomado en cuenta la terapia combinada de antiparasitarios en los protocolos de atención a la población infantil.

Recibido: 22 de enero de 2001.

Aceptado: 14 de agosto de 2001.

Los helmintos y protozoarios son parásitos intestinales de gran impacto en la salud de muchas poblaciones alrededor del mundo. Al estudiarlos hay que tener en cuenta aspectos tales como su distribución, por ejemplo, los helmintos Enterobius vermicularis, Trichuris trichiura, Ascaris lumbricoides se encuentran más frecuentemente en zonas templadas y tropicales; el Strongyloides stercoralis se encuentra en trópicos y subtrópicos húmedos. Los protozoarios intestinales como Entamoeba histolytica y Giardia lamblia se pueden encontrar en cualquier zona a nivel mundial. ${ }^{1-3}$

\footnotetext{
${ }^{1}$ Area de Salud, San Ramón de Alajuela.

${ }^{2}$ Area de Salud, Huetar Atlántica.
}

Correspondencia: Erasmo Serrano Frago. Correo electrónico: easfrago@ yahoo.com
Otros aspectos a conocer son sus ciclos de vida y su mecanismo de transmisión. En cuanto a los ciclos de vida; los helmintos son gusanos multicelulares que solo en raras ocasiones se pueden multiplicar en el ser humano, no así los protozoarios que al ser unicelulares pueden multiplicarse en este. Estos ciclos pueden ser simples o con huéspedes intermediarios como en el caso de la Taenia solium. ${ }^{1-3}$

El recibir en las consultas un alto porcentaje de pacientes con cuadros de parasitosis, motivó a realizar este estudio para conocer cual de las parasitosis ya mencionadas representa un índice de mayor frecuencia en la zona.

Al tener mayor frecuencia de infestación por protozoarios en la población infantil, podemos relacionar esto tanto al mecanismo de transmisión, fecal-oral o vía cutánea, como al descuido en las medidas higiénicas básicas; por lo tanto su tratamiento debe ser integral, farmacológico e incluir las medidas generales sobre la higiene que se deben tener. 
La educación continua a las comunidades y al personal de salud sobre el incremento en las parasitosis por protozoarios en nuestro medio, debe enfocarse a descuidos tan elementales como: la escasa o falta de cloración de las aguas, la eliminación indiscriminada de desechos orgánicos e inorgánicos a nuestras fuentes acuíferas, ocasionando un incremento en la contaminación de nuestro bioecosistema, y agravando problemas de salud en nuestras comunidades.

Las estrategías nacionales para la prevención y tratamiento de las parasitosis intestinales contempladas en las normas nacionales de atención integral de la salud han cumplido un gran papel en el tratamiento de la población infantil del país; sin embargo, en lo visto en este estudio, el aumento en las parasitosis por protozoarios, llevaría ha adoptar otras formas terapeuticas para su prevencion.

\section{Materiales y Métodos}

El presente estudio corresponde al área de salud de San Ramón de Alajuela, sobre una población estimada de 18002 niños de ambos sexos, comprendidos entre 1 y 12 años de edad, de donde se tomó una muestra retrospectiva de 5250 pacientes, a los que se les había enviado pruebas de laboratorio por sus médicos tratantes, por sospecha de parasitosis intestinal. Esta muestra representa un $29,16 \%$ de la población infantil de la zona y fue captada en la consulta externa de los diferentes puestos de salud en un período de un año que va de diciembre de 1999 a noviembre del 2000.

A la muestra se le realizó frotis y extendidos simples de heces con la técnica de solución salina y lugol, extrayendo así los agentes parasitarios más comunes.

Para tal efecto se creó un protocolo en el que se registraron las siguientes variables: edad, sexo, tipo de parásito, servicios básicos (agua de consumo humano, forma de eliminación de excretas y tipo de vivienda ) y zona de procedencia,

\begin{tabular}{|c|c|c|c|}
\hline \multicolumn{4}{|c|}{$\begin{array}{l}\text { Cuadro } 1 \\
\text { Distribución de la población estudiada según } \\
\text { resultado de la muestra de heces y grupo etario } \\
\text { Área de Salud de San Ramón de Alajuela, } \\
\text { diciembre } 1999 \text { a noviembre, } 2000\end{array}$} \\
\hline & $\begin{array}{c}\text { No infectados } \\
\quad N^{\circ}(\%)\end{array}$ & $\begin{array}{l}\text { Infectados } \\
\mathrm{N}^{\circ}(\%)\end{array}$ & $\begin{array}{l}\text { Total } \\
\mathrm{N}^{\circ}(\%)\end{array}$ \\
\hline 1-4 años & $1036(65.3)$ & $550(34.7)$ & $1586(100)$ \\
\hline 5-8 años & $1090(47.8)$ & 1190 (52.2) & $2280(100)$ \\
\hline 9-12 años & 1001 (72.3) & $383(27.7)$ & $1384(100)$ \\
\hline Total & 3127 (59.6) & $2123(40.4)$ & $5250(100)$ \\
\hline
\end{tabular}

tomando en cuenta que a excepción del distrito central el cual es suburbano los doce restantes son rurales.

Con los datos recabados de los expedientes clínicos de consulta externa, se realizó una revisión retrospectiva de la frecuencia con que los individuos sufrían de parasitosis en esta zona.

\section{Resultados}

Según la revisión de los expedientes y los resultados de laboratorio, se observó que existía una marcada tendencia en la muestra a presentar índices superiores de parasitosis por protozoarios que por helmintos. En cuanto al rango de edad, el grupo etario que presentó mayor parasitosis se encuentra entre los 5 y 8 años, siendo los hombres el sexo con mayor frecuencia a razón de 1,7:1 con respecto a las mujeres.

De los 5250 pacientes estudiados durante un año desglosados en 2685 hombres y 2565 mujeres; solo 2123 niños $(40,4 \%)$ en general (1335 hombres y 788 mujeres) resultaron con infección parasitaria, encontrándose 1478 pacientes infectados por protozoarios $(69,62 \%)$ y 645 pacientes infectados por helmintos (30,38\%; Cuadro 1). El $67 \%$ de los pacientes con infección parasitaria correspondían a zonas netamente rurales del área.

Los distritos del cantón se agruparon por zonas geográficas mostrando un mayor porcentaje de pacientes infectados por parasitosis intestinales en las zonas norte y oeste del área de salud en mención (Cuadro 2).

Hubo una frecuencia de 1012 casos de protozoarios $(75,81 \%)$ y 323 casos de helmintos $(24,19 \%)$ en hombres. Y los resultados en mujeres fueron de 465 casos por protozoarios $(59,01 \%)$ contra 323 casos por helmintos (40,99\%; Gráfico 1$)$.

Entre los casos de protozoarios tanto en hombres como en mujeres se encontraron los siguientes parásitos en orden de frecuencia: Entamoeba histolytica con 360 casos reportados,
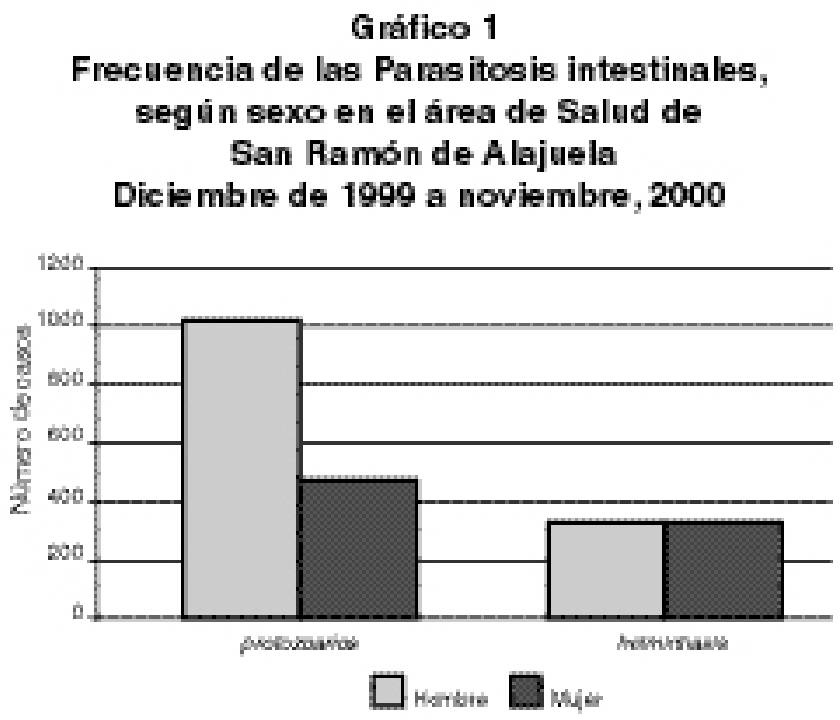


\begin{tabular}{|c|c|c|c|c|c|c|}
\hline \multicolumn{7}{|c|}{$\begin{array}{l}\text { Cuadro } 2 \\
\text { Distribución porcentual de pacientes infectados y no infectados, según división distrital por zonas } \\
\text { geográficas en el área de salud de San Ramón de Alajuela, diciembre de } 1999 \text { a noviembre de } 2000\end{array}$} \\
\hline \multirow{2}{*}{$\begin{array}{l}\text { Distribución por zonas } \\
\text { del cantón (distritos) }\end{array}$} & \multicolumn{2}{|c|}{ Pacientes no infectados } & \multicolumn{2}{|c|}{ Pacientes infectados } & \multicolumn{2}{|c|}{ Totales } \\
\hline & $\mathrm{N}^{\mathrm{O}}$ & $\%$ & $\mathrm{~N}^{\circ}$ & $\%$ & $\mathrm{~N}^{\circ}$ & $\%$ \\
\hline $\begin{array}{c}\text { Norte: } \\
\text { San Juan, Los Angeles, } \\
\text { Peñas Blancas }\end{array}$ & 843 & $53,4 \%$ & 734 & $46,5 \%$ & 1577 & $100 \%$ \\
\hline $\begin{array}{l}\text { Oeste: } \\
\text { Alfaro, Piedades norte y sur, } \\
\text { Zapotal }\end{array}$ & 758 & $54,1 \%$ & 643 & $45,9 \%$ & 1401 & $100 \%$ \\
\hline $\begin{array}{c}\text { Sur: } \\
\text { Central, San Rafael, Santiago }\end{array}$ & 707 & $61,9 \%$ & 435 & $38,1 \%$ & 1142 & $100 \%$ \\
\hline $\begin{array}{c}\text { Este: } \\
\text { Volio, San Isidro y Concepción }\end{array}$ & 819 & $72,5 \%$ & 311 & $27,5 \%$ & 1130 & $100 \%$ \\
\hline Totales & 3127 & $59,6 \%$ & 2123 & $40,4 \%$ & 5250 & $100 \%$ \\
\hline
\end{tabular}

Entamoeba coli con 338 casos reportados, Giardia lamblia con 293 casos reportados, Endolimax nana 263 casos reportados, Balantidiun coli 187 casos reportados, y otros con 37 casos reportados (Gráfico 2).

En el caso de los helmintos tanto en hombres como en mujeres los parásitos encontrados se muestran en el Gráfico 3; Enterobius vermicularis con 143 casos reportados, Strongyloides stercoralis con 120 casos reportados, Himenolepis nana con 90 casos reportados, Taenia solium 82 casos reportados, Ascaris lumbricoides con 68 casos reportados, Necator americanus 60 casos reportados, Trichurus trichiuris 52 casos reportados, y otros con 30 casos reportados.

Cabe destacar que el 98,5\% de la población consume agua potable suministrada por un acueducto rural la cual proviene de ríos y manantiales debidamente tratados, administrado por la municipalidad en algunos casos y por los comités de desarrollo comunal de cada distrito y el 1,5\% utiliza agua de pozos artesanales y ríos no tratados. Cabe destacar que en recientes valoraciones de calidad de agua de esta zona se encontró, que las aguas del área norte y oeste del cantón están contaminadas con residuos de materia fecal entre otros. Además están siendo utilizadas para el consumo humano.

La eliminación de excretas en un $64 \%$ se da en tanques sépticos, un $35 \%$ por letrinas y $1 \%$ otras formas. El tipo de vivienda que prevalece es la construcción mixta.

\section{Discusión}

En los últimos años los parásitos intestinales han sido uno de los mayores problemas de salud tanto en Costa Rica como en Latinoamérica. $^{4}$
Los helmintos que infectan al hombre se dividen en 2 grupos: nematelmintos o gusanos redondos que corresponden a los nemátodos y los platelmintos o gusanos planos que son tremátodos y céstodos, que afectan a una cuarta parte de la población mundial. La sintomatología presentada por ellos es tan variada que su estudio es de suma importancia. Entre los síntomas más comunes podemos mencionar; el prurito anal, dolor abdominal, perdida de peso, desnutrición, síndromes de mala absorción, fiebre, diarrea y en casos extremos la muerte. ${ }^{4,5}$

Los parásitos intestinales son más frecuentes en niños que en adultos y su mayor incidencia se da en poblaciones de baja condición económica, educativa y social, donde los servicios básicos como agua y servicios sanitarios entre otros, no exis-

\section{Gráfico 2}

Distribución de los protozoarios intestinales, según frecuencia del parásito, en el área de Salud de San Ramón de Alajuela Diciembre de 1999 a noviembre, 2000

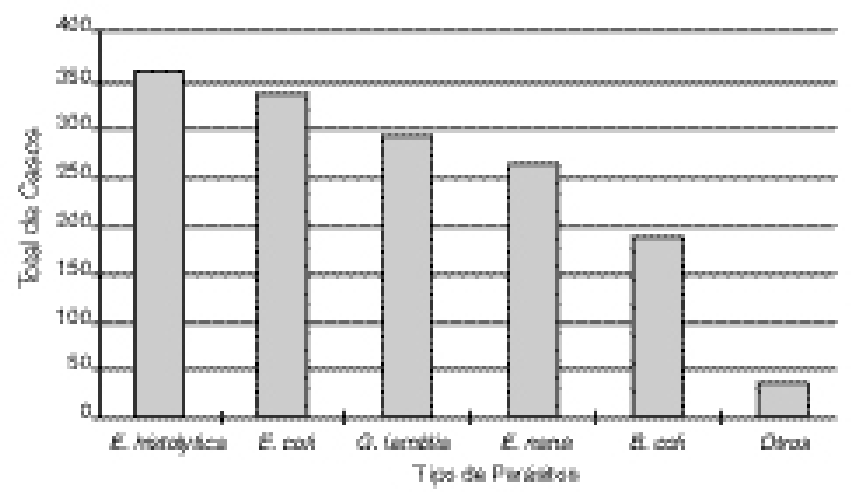




\section{Gráfico 3 \\ Distribución de la helmintiasis intestinal, según frecuencia del parásito, en el área de Salud de San Ramón de Alajuela \\ Diciembre de 1999 a nov iembre, 2000}

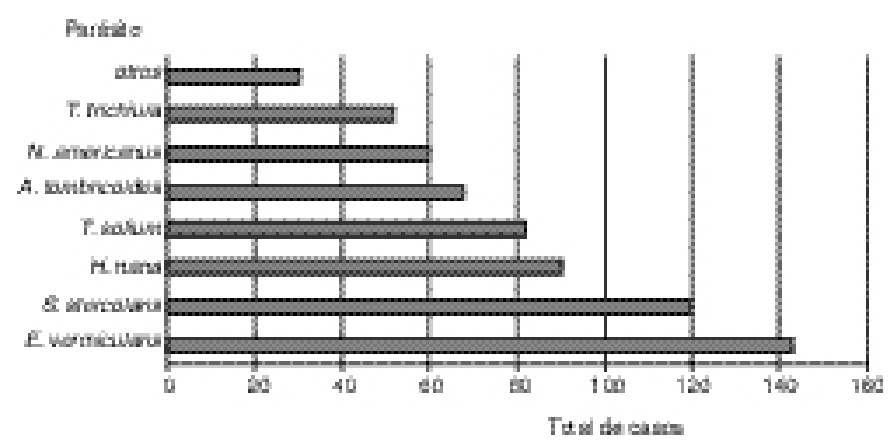

ten o están en deplorable estado. Es muy importante tomar esto en cuenta ya que el mecanismo de transmisión se da por contaminación ano-mano-boca, por la vía cutánea o por contaminación y mal manejo de alimentos o agua. ${ }^{6-8}$

Se observó una mayor frecuencia de casos reportados con protozoarios, con respecto a casos reportados con helmintiasis. Presentándose un mayor porcentaje de Entamoeba histolytica y coli, seguida por Endolimax nana, y Balantidium coli, en cuanto a los primeros y Enterobius vermicularis, Strongyloides stercoralis, Himenolepis nana y Ascaris lumbricoides en cuanto a los segundos; debido esto a que la zona estudiada se encuentra entre los parámetros descritos anteriormente, agrícola de pobres condiciones sanitarias y con una alta tasa de inmigrantes centroamericanos. ${ }^{9}$

En estudios realizados en Beni-Mellal, zona agrícola en la que se utiliza en muchas ocasiones agua no pura para los riegos, se ha observado que los parásitos, más frecuentes son Entamoeba histolytica, Giardia intestinalis, Ascaris lumbricoides, Trichurus trichiura, Enterobios vermicularis e Himenolopis nana. ${ }^{10}$

En Nicaragua existen estudios donde han asociado el grado de desnutrición con las parásitos y han encontrado Ascaris lumbricoides y Trichurus trichiuria como los parásitos de mayor frecuencia en niños desnutridos. ${ }^{11}$

Maracaibo, un estudio realizado a niños menores de 5 años encontró un mayor predominio de protozoarios (29\%) sobre los helmintos $(10 \%){ }^{12,13}$

En Costa Rica en el año 1996 se realizó un estudio en la población de Hatillo, encontrándose una mayor frecuencia por los siguientes parásitos Entamoeba coli, Endolimax nana, Giardia lamblia y Ascaris lumbricoides, al doble que en años anteriores. En otro estudio realizado en las poblaciones de Acosta y Coronado en el año 1997 se observó la misma frecuencia anterior. ${ }^{14,15}$
Parte muy importante en la lucha de los parásitos, es el tratamiento que debe constituirse en dos partes; una de ellas y la más importante es la educación básica en la población como por ejemplo: lavado de las manos después de usar el servicio sanitario, lavar las frutas y vegetales con agua potable, mantener buen aseo personal, buen manejo de excretas y uso de calzado entre otras, para evitar los focos y contactos de contaminación. ${ }^{9,16,17}$

El otro, es el tratamiento farmacológico de uso profiláctico en niños de edad temprana, este es una de las mejores estrategias en la lucha contra las helmintiasis, existen combinaciones de drogas que han demostrado aumentar la eficacia de ciertos antiparasitarios por ejemplo; al combinar cimetidina con mebendazol, ya que se aumenta la concentración sérica de éste último. Durante el embarazo, uno de los mayores problemas de las parasitosis, es el elevado riesgo a realizar anemias por déficit de hierro. Se recomienda el uso de metronidazol después del primer trimestre de embarazo ya que se ha reportado un caso de malformación a causa de este y además es teratogénico en ratas. El albendazol se absorbe en mayores cantidades que el mebendazol por lo que es posible asociarlo a mayores efectos adversos por lo que su uso no es recomendado en el embarazo. El praziquantel a pesar de que no se ha encontrado teratogenia en animales. ${ }^{18-21}$

Se encontró en la población estudiada la existencia de una mayor tendencia hacia las parasitosis intestinales por protozoarios que las causadas por helmintos, semejante a estudios realizados en otros países.

En cuanto a los rangos etarios la incidencia de parasitosis se dio entre los tres y los ocho años de edad, lo cual se ajusta a los valores mundiales sobre infecciones parasitarias; tomando en cuenta que los hombres son la población más afectada.

Por otro lado, la tendencia a la parasitosis por helmintos en esta zona, es relativamente menor en comparación con los datos nacionales de años atrás. Actualmente estos datos se encuentran invertidos, debido al advenimiento de la desparasitación masiva a nivel de centros educativos y de la población en general con antihelmínticos.

Los problemas socioeconómicos tales como la inmigración, los niveles de desnutrición, el desempleo y el hacinamiento, entre otros son los principales causantes de las parasitosis a nivel local; al mejorar dichas situaciones mediante la educación y la higiene de la población se puede minimizar los riesgos a la salud pública.

Los altos niveles de contaminación encontrados en las diferentes fuentes de agua para consumo humano en la zona norte y oeste del cantón, podría tener una marcada relación con la alta frecuencia de parasitosis intestinales obtenidas en este estudio.

Se ha podido encontrar que el uso combinado en la terapia antiparasitaria, no solo como tratamiento sino como profilaxis sería lo mas recomendado para el manejo de las poblaciones infantiles de nuestro país. 


\section{Abstract}

Background and aim: Intestinal parasitosis by protozoa and helminths, remains a public health problem in many places around the globe. The present study aimes to determine the frequency of these parasitosis in children between 1 and 12 years old in San Ramón, Alajuela, Costa Rica.

Method: In this retrospective study 5250 fecal samples of children between 1 and 12 years old were studied. The samples were collected and examined from December 1999 to November 2000. The samples were ordered by the treating physician, in patients whom they suspected as having intestinal parasitosis. The information was obtained from the medical files of each patient.

Results: Of the 5250 cases studied $40.4 \%$ had parasites in the fecal sample. A majority of the cases corresponded to protozoa (1478 samples), and 645 samples were positive for helminths. Among the protozoa found the most frequent ones were E. histolytica, E. coli y G. intestinalis. Among the helminths $E$. vernicularis, $S$. stercoralis and $H$. nana were the most frequent ones. With regard to sex distribution $30.7 \%$ of the girls had parasitosis compared to $49.7 \%$ of the boys.

Conclusion: Intestinal parasitic infection remains highly frequent among the pediatric population. There has been a reduction in the frequency of helminths due to massive control campaigns. However, protozoa have emerged due to a lack of combined antiparasitic therapy in these campaigns.

\section{Referencias}

1. Isselbacher K, Braunwald E, Wilson J, Martin J, Fauci A, Kasper D. Principios de Medicina Interna. México: MacGraw-Hill, 1994; 10641068.

2. Huertas A. Fundamentos de la parasitología clinica.Costa Rica: EDUNED, 1984; 62-152.

3. Wyngaarden J, Smith L Tratado de Medicina Interna. México: MacGraw-Hill, 1991: 2045-2116.

4. Ananthakrishnan S, Nalini P, Pani SP. Intestinal geohelminthiasis in the developing world. Natl Med J India 1997; 10: 67-71.

5. Crompton DW, Savioli L. Intestinal parasitic infections and urbanization. Bull World Health Organ 1993; 71:1-7.

6. Dieng Y, Tandia AA, Wane AT, Gaye O, Diop EH, Diallo S. Intestinal parasitosis in the inhabitants of a suburban zone in which the groundwater is polluted by nitrates of fecal origin (Yeumbeul, Senegal). Sante; 1999; 9: 351-6-.

7. Jarabo MT, Garcia-Moran NP, Garcia-Moran JI. Prevalence of intestinal parasites in a student population. Enferm Infecc Microbiol Clin 1995; 13: 464-8.

8. Albonico M, Savioli L. Hookworm infection and disease: advances for control. Ann Ist Super Sanita 1997; 33: 567-79.

9. Muennig P, Pallin D, Sell RL, Chan MS. The cost effectiveness of strategies for the treatment of intestinal parasites in immigrants. N Engl J Med 1999; 11; 340: 773-9.
10. Habbari K, Tifnouti A, Bitton G, Mandil. A. Intestinal parasitosis and environmental pollution: 1343 pediatric cases in Beni-Mellal, Morocco. Tunis Med 2000; 78: 109-14.

11. Oberhelman RA, Guerrero ES, Fernandez ML, Silio M, Mercado D. Comiskey, N; Ihenacho, G, Mera, R. Correlations between intestinal parasitosis, physical growth, and psychomotor development among infants and children from rural Nicaragua. Am J Trop Med Hyg 1998; 58: 470-5.

12. Rincón de Heredia W, Calvo B, Acurero E, Chaparro O, Paz M, Guanipa $\mathrm{S}$, Heredia M. Prevalencia de parásitos intestinales en niños menores de 5 años con diarrea a tendidos en centros asistenciales de la Ciudad de Maracaibo. Kasmera, 1995; 23: 27-41.

13. Díaz I, Flores T. Prevalencia de parásitos intestinales en alumnos de educación básica del municipio Cacique Mara, Maracaibo Estado Zulia. Kasmera;1990; 18: 46-70.

14. Pardo V, Hernández F. Prevalencia de parásitos intestinales en una población atendida en la Clínica de Hatillo del Ministerio de Salud, 1995-1996. Rev costarric cienc med 1997 18: 45-50.

15. Bolaños N, Guevara A, Free E. Prevalencia de parásitos intestinales en las áreas de salud de Acosta y Coronado. Rev costarric cienc med 1997, 18: 41-9.

16. Stephenson I, Wiselka M. Drug treatment of tropical parasitic infections: recent achievements and developments. Drugs 2000; 60: 98595 .

17. Ludwig KM, Frei F, Alvares Filho F, Ribeiro-Paes JT. Correlation between sanitation conditions and intestinal parasitosis in the population of Assis, State of Sao. Rev Soc Bras Med Trop 1999; 32: 547-55.

18. Bennett A, Guyatt $H$. Reducing intestinal nematode infection: efficacy of albendazole and mebendazole. Parasitol Today 2000;16: 71-4.

19. Vande Waa E, Herdenson J, White G, Nowatcke T. Anthelmintic: 5 agentes cover the most common parasitic worn infections. Drugs and Therapy perspectives. 1998, 11: 9-13.

20. Borda CE, Rea MJ, Rosa JR, Maidana C. Intestinal parasitism in San Cayetano, Corrientes, Argentina. Bull Pan Am Health Organ 1996; 30: 227-33.

21. Wakelin D. Immune responses to intestinal parasites: protection, pathology and prophylaxis. Parassitologia; 1997; 39: 269-74. 